\title{
Ostrowski and Trapezoid Type Inequalities for the Generalized $k=g$-Fractional Integrals of Functions with Bounded Variation
}

\author{
Silvestru Sever Dragomir ${ }^{1 *}$
}

\begin{abstract}
In this paper we establish some Ostrowski and trapezoid type inequalities for the $k$ - $g$-fractional integrals of functions of bounded variation. Applications for mid-point and trapezoid inequalities are provided as well. Some examples for a general exponential fractional integral are also given.

Keywords: Functions of bounded variation, Generalized Riemann-Liouville fractional integrals, Hadamard fractional integrals, Ostrowski type inequalities, Trapezoid inequalities

2010 AMS: 26D15, 26D10, 26D07, 26A33

${ }^{1}$ Mathematics, College of Engineering \& Science Victoria University, Melbourne City, MC 8001, Australia, ORCID: 0000-0003-2902-6805

*Corresponding author: sever.dragomir@vu.edu.au

Received: 02 October 2019, Accepted: 21 November 2019, Available online: 29 December 2019
\end{abstract}

\section{Introduction}

Assume that the kernel $k$ is defined either on $(0, \infty)$ or on $[0, \infty)$ with complex values and integrable on any finite subinterval. We define the function $K:[0, \infty) \rightarrow \mathbb{C}$ by

$$
K(t):=\left\{\begin{array}{l}
\int_{0}^{t} k(s) d s \text { if } 0<t \\
0 \text { if } t=0
\end{array}\right.
$$

As a simple example, if $k(t)=t^{\alpha-1}$ then for $\alpha \in(0,1)$ the function $k$ is defined on $(0, \infty)$ and $K(t):=\frac{1}{\alpha} t^{\alpha}$ for $t \in[0, \infty)$. If $\alpha \geq 1$, then $k$ is defined on $[0, \infty)$ and $K(t):=\frac{1}{\alpha} t^{\alpha}$ for $t \in[0, \infty)$.

Let $g$ be a strictly increasing function on $(a, b)$, having a continuous derivative $g^{\prime}$ on $(a, b)$. For the Lebesgue integrable function $f:(a, b) \rightarrow \mathbb{C}$, we define the $k$-g-left-sided fractional integral of $f$ by

$$
S_{k, g, a+} f(x)=\int_{a}^{x} k(g(x)-g(t)) g^{\prime}(t) f(t) d t, x \in(a, b]
$$

and the $k$-g-right-sided fractional integral of $f$ by

$$
S_{k, g, b-} f(x)=\int_{x}^{b} k(g(t)-g(x)) g^{\prime}(t) f(t) d t, x \in[a, b) .
$$


If we take $k(t)=\frac{1}{\Gamma(\alpha)} t^{\alpha-1}$, where $\Gamma$ is the Gamma function, then

$$
\begin{aligned}
S_{k, g, a+} f(x) & =\frac{1}{\Gamma(\alpha)} \int_{a}^{x}[g(x)-g(t)]^{\alpha-1} g^{\prime}(t) f(t) d t \\
& =: I_{a+, g}^{\alpha} f(x), a<x \leq b
\end{aligned}
$$

and

$$
\begin{aligned}
S_{k, g, b-} f(x) & =\frac{1}{\Gamma(\alpha)} \int_{x}^{b}[g(t)-g(x)]^{\alpha-1} g^{\prime}(t) f(t) d t \\
& =: I_{b-, g}^{\alpha} f(x), a \leq x<b,
\end{aligned}
$$

which are the generalized left- and right-sided Riemann-Liouville fractional integrals of a function $f$ with respect to another function $g$ on $[a, b]$ as defined in $[1, \mathrm{p} .100]$

For $g(t)=t$ in (1.3) we have the classical Riemann-Liouville fractional integrals while for the logarithmic function $g(t)=\ln t$ we have the Hadamard fractional integrals [1, p. 111]

$$
H_{a+}^{\alpha} f(x):=\frac{1}{\Gamma(\alpha)} \int_{a}^{x}\left[\ln \left(\frac{x}{t}\right)\right]^{\alpha-1} \frac{f(t) d t}{t}, 0 \leq a<x \leq b
$$

and

$$
H_{b-}^{\alpha} f(x):=\frac{1}{\Gamma(\alpha)} \int_{x}^{b}\left[\ln \left(\frac{t}{x}\right)\right]^{\alpha-1} \frac{f(t) d t}{t}, 0 \leq a<x<b .
$$

One can consider the function $g(t)=-t^{-1}$ and define the "Harmonic fractional integrals" by

$$
R_{a+}^{\alpha} f(x):=\frac{x^{1-\alpha}}{\Gamma(\alpha)} \int_{a}^{x} \frac{f(t) d t}{(x-t)^{1-\alpha} t^{\alpha+1}}, 0 \leq a<x \leq b
$$

and

$$
R_{b-}^{\alpha} f(x):=\frac{x^{1-\alpha}}{\Gamma(\alpha)} \int_{x}^{b} \frac{f(t) d t}{(t-x)^{1-\alpha} t^{\alpha+1}}, 0 \leq a<x<b .
$$

Also, for $g(t)=\exp (\beta t), \beta>0$, we can consider the " $\beta$-Exponential fractional integrals"

$$
E_{a+, \beta}^{\alpha} f(x):=\frac{\beta}{\Gamma(\alpha)} \int_{a}^{x}[\exp (\beta x)-\exp (\beta t)]^{\alpha-1} \exp (\beta t) f(t) d t
$$

for $a<x \leq b$ and

$$
E_{b-, \beta}^{\alpha} f(x):=\frac{\beta}{\Gamma(\alpha)} \int_{x}^{b}[\exp (\beta t)-\exp (\beta x)]^{\alpha-1} \exp (\beta t) f(t) d t
$$

for $a \leq x<b$.

If we take $g(t)=t$ in (1.1) and (1.2), then we can consider the following $k$-fractional integrals

$$
S_{k, a+} f(x)=\int_{a}^{x} k(x-t) f(t) d t, x \in(a, b]
$$

and

$$
S_{k, b-} f(x)=\int_{x}^{b} k(t-x) f(t) d t, x \in[a, b) .
$$

In [2], Raina studied a class of functions defined formally by

$$
\mathscr{F}_{\rho, \lambda}^{\sigma}(x):=\sum_{k=0}^{\infty} \frac{\sigma(k)}{\Gamma(\rho k+\lambda)} x^{k},|x|<R, \text { with } R>0
$$


for $\rho, \lambda>0$ where the coefficients $\sigma(k)$ generate a bounded sequence of positive real numbers. With the help of (1.6), Raina defined the following left-sided fractional integral operator

$$
\mathscr{J}_{\rho, \lambda, a+; w}^{\sigma} f(x):=\int_{a}^{x}(x-t)^{\lambda-1} \mathscr{F}_{\rho, \lambda}^{\sigma}\left(w(x-t)^{\rho}\right) f(t) d t, x>a
$$

where $\rho, \lambda>0, w \in \mathbb{R}$ and $f$ is such that the integral on the right side exists.

In [3], the right-sided fractional operator was also introduced as

$$
\mathscr{J}_{\rho, \lambda, b-; w}^{\sigma} f(x):=\int_{x}^{b}(t-x)^{\lambda-1} \mathscr{F}_{\rho, \lambda}^{\sigma}\left(w(t-x)^{\rho}\right) f(t) d t, x<b
$$

where $\rho, \lambda>0, w \in \mathbb{R}$ and $f$ is such that the integral on the right side exists. Several Ostrowski type inequalities were also established.

We observe that for $k(t)=t^{\lambda-1} \mathscr{F}_{\rho, \lambda}^{\sigma}\left(w t^{\rho}\right)$ we re-obtain the definitions of (1.7) and (1.8) from (1.4) and (1.5).

In [4], Kirane and Torebek introduced the following exponential fractional integrals

$$
\mathscr{T}_{a+}^{\alpha} f(x):=\frac{1}{\alpha} \int_{a}^{x} \exp \left\{-\frac{1-\alpha}{\alpha}(x-t)\right\} f(t) d t, x>a
$$

and

$$
\mathscr{T}_{b-}^{\alpha} f(x):=\frac{1}{\alpha} \int_{x}^{b} \exp \left\{-\frac{1-\alpha}{\alpha}(t-x)\right\} f(t) d t, x<b
$$

where $\alpha \in(0,1)$.

We observe that for $k(t)=\frac{1}{\alpha} \exp \left(-\frac{1-\alpha}{\alpha} t\right), t \in \mathbb{R}$ we re-obtain the definitions of (1.9) and (1.10) from (1.4) and (1.5).

Let $g$ be a strictly increasing function on $(a, b)$, having a continuous derivative $g^{\prime}$ on $(a, b)$. We can define the more general exponential fractional integrals

$$
\mathscr{T}_{g, a+}^{\alpha} f(x):=\frac{1}{\alpha} \int_{a}^{x} \exp \left\{-\frac{1-\alpha}{\alpha}(g(x)-g(t))\right\} g^{\prime}(t) f(t) d t, x>a
$$

and

$$
\mathscr{T}_{g, b-}^{\alpha} f(x):=\frac{1}{\alpha} \int_{x}^{b} \exp \left\{-\frac{1-\alpha}{\alpha}(g(t)-g(x))\right\} g^{\prime}(t) f(t) d t, x<b
$$

where $\alpha \in(0,1)$.

Let $g$ be a strictly increasing function on $(a, b)$, having a continuous derivative $g^{\prime}$ on $(a, b)$. Assume that $\alpha>0$. We can also define the logarithmic fractional integrals

$$
\mathscr{L}_{g, a+}^{\alpha} f(x):=\int_{a}^{x}(g(x)-g(t))^{\alpha-1} \ln (g(x)-g(t)) g^{\prime}(t) f(t) d t,
$$

for $0<a<x \leq b$ and

$$
\mathscr{L}_{g, b-}^{\alpha} f(x):=\int_{x}^{b}(g(t)-g(x))^{\alpha-1} \ln (g(t)-g(x)) g^{\prime}(t) f(t) d t,
$$

for $0<a \leq x<b$, where $\alpha>0$. These are obtained from (1.4) and (1.5) for the kernel $k(t)=t^{\alpha-1} \ln t, t>0$.

For $\alpha=1$ we get

$$
\mathscr{L}_{g, a+} f(x):=\int_{a}^{x} \ln (g(x)-g(t)) g^{\prime}(t) f(t) d t, 0<a<x \leq b
$$

and

$$
\mathscr{L}_{g, b-} f(x):=\int_{x}^{b} \ln (g(t)-g(x)) g^{\prime}(t) f(t) d t, 0<a \leq x<b .
$$


For $g(t)=t$, we have the simple forms

$$
\begin{aligned}
& \mathscr{L}_{a+}^{\alpha} f(x):=\int_{a}^{x}(x-t)^{\alpha-1} \ln (x-t) f(t) d t, 0<a<x \leq b, \\
& \mathscr{L}_{b-}^{\alpha} f(x):=\int_{x}^{b}(t-x)^{\alpha-1} \ln (t-x) f(t) d t, 0<a \leq x<b, \\
& \mathscr{L}_{a+} f(x):=\int_{a}^{x} \ln (x-t) f(t) d t, 0<a<x \leq b
\end{aligned}
$$

and

$$
\mathscr{L}_{b-} f(x):=\int_{x}^{b} \ln (t-x) f(t) d t, 0<a \leq x<b .
$$

In the recent paper [5] we obtained the following Ostrowski and trapezoid type inequalities for the generalized left- and right-sided Riemann-Liouville fractional integrals of a function $f$ with respect to another function $g$ on $[a, b]$.

Theorem 1.1. Let $f:[a, b] \rightarrow \mathbb{C}$ be a function of bounded variation on $[a, b]$. Also let $g$ be a strictly increasing function on $(a, b)$, having a continuous derivative $g^{\prime}$ on $(a, b)$. Then we have

$$
\begin{aligned}
& \left|I_{x-, g}^{\alpha} f(a)+I_{x+, g}^{\alpha} f(b)-\frac{1}{\Gamma(\alpha+1)}\left[(g(x)-g(a))^{\alpha}+(g(b)-g(x))^{\alpha}\right] f(x)\right| \\
& \leq \frac{1}{\Gamma(\alpha)}\left[\int_{a}^{x}(g(t)-g(a))^{\alpha-1} g^{\prime}(t) \bigvee_{t}^{x}(f) d t+\int_{x}^{b}(g(b)-g(t))^{\alpha-1} g^{\prime}(t) \bigvee_{x}^{t}(f) d t\right] \\
& \leq \frac{1}{\Gamma(\alpha+1)}\left[\begin{array}{l}
\left.(g(x)-g(a))^{\alpha} \bigvee_{a}^{x}(f)+(g(b)-g(x))^{\alpha} \bigvee_{x}^{b}(f)\right] \\
\left((g(x)-g(a))^{\alpha p}+(g(b)-g(x))^{\alpha p}\right)^{1 / p}\left(\left(\bigvee_{a}^{x}(f)\right)^{q}+\left(\bigvee_{x}^{b}(f)\right)^{q}\right)^{1 / q} \\
\text { with } p, q>1, \frac{1}{p}+\frac{1}{q}=1 ; \\
{\left[\frac{1}{2}(g(b)-g(a))+\left|g(x)-\frac{g(a)+g(b)}{2}\right|\right]^{\alpha} \bigvee_{a}^{b}(f) ;}
\end{array}\right. \\
& \leq \frac{1}{\Gamma(\alpha+1)}\left\{\begin{array}{l}
\left.[f)+\frac{1}{2}\left|\bigvee_{a}^{x}(f)-\bigvee_{x}^{b}(f)\right|\right]\left((g(x)-g(a))^{\alpha}+(g(b)-g(x))^{\alpha}\right)
\end{array}\right.
\end{aligned}
$$

and

$$
\begin{aligned}
& \left|I_{a+, g}^{\alpha} f(x)+I_{b-, g}^{\alpha} f(x)-\frac{1}{\Gamma(\alpha+1)}\left[(g(x)-g(a))^{\alpha} f(a)+(g(b)-g(x))^{\alpha} f(b)\right]\right| \\
& \leq \frac{1}{\Gamma(\alpha)}\left[\int_{a}^{x}(g(x)-g(t))^{\alpha-1} g^{\prime}(t) \bigvee_{a}^{t}(f) d t+\int_{x}^{b}(g(t)-g(x))^{\alpha-1} g^{\prime}(t) \bigvee_{t}^{b}(f) d t\right] \\
& \leq \frac{1}{\Gamma(\alpha+1)}\left[\begin{array}{l}
\left.(g(x)-g(a))^{\alpha} \bigvee_{a}^{x}(f)+(g(b)-g(x))^{\alpha} \bigvee_{x}^{b}(f)\right] \\
\left((g(x)-g(a))^{\alpha p}+(g(b)-g(x))^{\alpha p}\right)^{1 / p}\left(\left(\bigvee_{a}^{x}(f)\right)^{q}+\left(\bigvee_{x}^{b}(f)\right)^{q}\right)^{1 / q}
\end{array}\right. \\
& \leq \frac{1}{\Gamma(\alpha+1)}\left\{\begin{array}{l}
{\left[\frac{1}{2}(g(b)-g(a))+\left|g(x)-\frac{g(a)+g(b)}{2}\right|\right]^{\alpha} \bigvee_{a}^{b}(f) ;} \\
\text { with } p, q>1, \frac{1}{p}+\frac{1}{q}=1 ; \\
{\left[\frac{1}{2} \bigvee_{a}^{b}(f)+\frac{1}{2}\left|\bigvee_{a}^{x}(f)-\bigvee_{x}^{b}(f)\right|\right]\left((g(x)-g(a))^{\alpha}+(g(b)-g(x))^{\alpha}\right)}
\end{array}\right.
\end{aligned}
$$

for any $x \in(a, b)$. 
For applications to the classical Riemann-Liouville fractional integrals, Hadamard fractional integrals and Harmonic fractional integrals see [5].

For several Ostrowski type inequalities for Riemann-Liouville fractional integrals see [6]-[21], [22]-[32] and the references therein.

Motivated by the above results, in this paper we establish some Ostrowski and trapezoid type inequalities for the $k$ - $g$ fractional integrals of functions of bounded variation. Applications for mid-point and trapezoid inequalities are provided as well. Some examples for a general exponential fractional integral are also given.

\section{Some identities for the operator $S_{k, g, a+, b-}$}

For $k$ and $g$ as at the beginning of Introduction, we consider the mixed operator

$$
\begin{aligned}
& S_{k, g, a+, b-} f(x) \\
& :=\frac{1}{2}\left[S_{k, g, a+} f(x)+S_{k, g, b-} f(x)\right] \\
& =\frac{1}{2}\left[\int_{a}^{x} k(g(x)-g(t)) g^{\prime}(t) f(t) d t+\int_{x}^{b} k(g(t)-g(x)) g^{\prime}(t) f(t) d t\right]
\end{aligned}
$$

for the Lebesgue integrable function $f:(a, b) \rightarrow \mathbb{C}$ and $x \in(a, b)$.

The following two parameters representation for the operator $S_{k, g, a+, b-}$ holds:

Lemma 2.1. With the above assumptions for $k, g$ and $f$ we have

$$
\begin{aligned}
S_{k, g, a+, b-} f(x) & =\frac{1}{2}[\gamma K(g(b)-g(x))+\lambda K(g(x)-g(a))] \\
& +\frac{1}{2} \int_{a}^{x} k(g(x)-g(t)) g^{\prime}(t)[f(t)-\lambda] d t \\
& +\frac{1}{2} \int_{x}^{b} k(g(t)-g(x)) g^{\prime}(t)[f(t)-\gamma] d t
\end{aligned}
$$

for any $\lambda, \gamma \in \mathbb{C}$.

Proof. We have, by taking the derivative over $t$ and using the chain rule, that

$$
[K(g(x)-g(t))]^{\prime}=K^{\prime}(g(x)-g(t))(g(x)-g(t))^{\prime}=-k(g(x)-g(t)) g^{\prime}(t)
$$

for $t \in(a, x)$ and

$$
[K(g(t)-g(x))]^{\prime}=K^{\prime}(g(t)-g(x))(g(t)-g(x))^{\prime}=k(g(t)-g(x)) g^{\prime}(t)
$$

for $t \in(x, b)$.

Therefore, for any $\lambda, \gamma \in \mathbb{C}$ we have

$$
\begin{aligned}
& \int_{a}^{x} k(g(x)-g(t)) g^{\prime}(t)[f(t)-\lambda] d t \\
& =\int_{a}^{x} k(g(x)-g(t)) g^{\prime}(t) f(t) d t-\lambda \int_{a}^{x} k(g(x)-g(t)) g^{\prime}(t) d t \\
& =S_{k, g, a+} f(x)+\lambda \int_{a}^{x}[K(g(x)-g(t))]^{\prime} d t \\
& =S_{k, g, a+} f(x)+\left.\lambda[K(g(x)-g(t))]\right|_{a} ^{x}=S_{k, g, a+} f(x)-\lambda K(g(x)-g(a))
\end{aligned}
$$

and

$$
\begin{aligned}
& \int_{x}^{b} k(g(t)-g(x)) g^{\prime}(t)[f(t)-\gamma] d t \\
& =\int_{x}^{b} k(g(t)-g(x)) g^{\prime}(t) f(t) d t-\gamma \int_{x}^{b} k(g(t)-g(x)) g^{\prime}(t) d t \\
& =S_{k, g, b-} f(x)-\gamma \int_{x}^{b}[K(g(t)-g(x))]^{\prime} d t \\
& =S_{k, g, b-} f(x)-\left.\gamma[K(g(t)-g(x))]\right|_{x} ^{b}=S_{k, g, b-} f(x)-\gamma K(g(b)-g(x))
\end{aligned}
$$


for $x \in(a, b)$.

If we add the equalities (2.2) and (2.3) and divide by 2 then we get the desired result (2.1).

Corollary 2.2. With the above assumptions for $k, g$ and $f$ we have the Ostrowski type identity

$$
\begin{aligned}
S_{k, g, a+, b-} f(x) & =\frac{1}{2}[K(g(b)-g(x))+K(g(x)-g(a))] f(x) \\
& +\frac{1}{2} \int_{a}^{x} k(g(x)-g(t)) g^{\prime}(t)[f(t)-f(x)] d t \\
& +\frac{1}{2} \int_{x}^{b} k(g(t)-g(x)) g^{\prime}(t)[f(t)-f(x)] d t
\end{aligned}
$$

and the trapezoid type identity

$$
\begin{aligned}
S_{k, g, a+, b-} f(x) & =\frac{1}{2}[K(g(b)-g(x)) f(b)+K(g(x)-g(a)) f(a)] \\
& +\frac{1}{2} \int_{a}^{x} k(g(x)-g(t)) g^{\prime}(t)[f(t)-f(a)] d t \\
& +\frac{1}{2} \int_{x}^{b} k(g(t)-g(x)) g^{\prime}(t)[f(t)-f(b)] d t
\end{aligned}
$$

for any $x \in(a, b)$.

For $x=\frac{a+b}{2}$ we can consider

$$
\begin{aligned}
M_{k, g, a+, b-} f & :=S_{k, g, a+, b-} f\left(\frac{a+b}{2}\right) \\
& =\frac{1}{2} \int_{a}^{\frac{a+b}{2}} k\left(g\left(\frac{a+b}{2}\right)-g(t)\right) g^{\prime}(t) f(t) d t \\
& +\frac{1}{2} \int_{\frac{a+b}{2}}^{b} k\left(g(t)-g\left(\frac{a+b}{2}\right)\right) g^{\prime}(t) f(t) d t .
\end{aligned}
$$

By (2.4) we have the representation

$$
\begin{aligned}
& M_{k, g, a+, b-} f \\
& =\frac{1}{2}\left[K\left(g(b)-g\left(\frac{a+b}{2}\right)\right)+K\left(g\left(\frac{a+b}{2}\right)-g(a)\right)\right] f\left(\frac{a+b}{2}\right) \\
& +\frac{1}{2} \int_{a}^{\frac{a+b}{2}} k\left(g\left(\frac{a+b}{2}\right)-g(t)\right) g^{\prime}(t)\left[f(t)-f\left(\frac{a+b}{2}\right)\right] d t \\
& +\frac{1}{2} \int_{\frac{a+b}{2}}^{b} k\left(g(t)-g\left(\frac{a+b}{2}\right)\right) g^{\prime}(t)\left[f(t)-f\left(\frac{a+b}{2}\right)\right] d t
\end{aligned}
$$

and (2.5) we have

$$
\begin{aligned}
& M_{k, g, a+, b-} f \\
& =\frac{1}{2}\left[K\left(g(b)-g\left(\frac{a+b}{2}\right)\right) f(b)+K\left(g\left(\frac{a+b}{2}\right)-g(a)\right) f(a)\right] \\
& +\frac{1}{2} \int_{a}^{\frac{a+b}{2}} k\left(g\left(\frac{a+b}{2}\right)-g(t)\right) g^{\prime}(t)[f(t)-f(a)] d t \\
& +\frac{1}{2} \int_{\frac{a+b}{2}}^{b} k\left(g(t)-g\left(\frac{a+b}{2}\right)\right) g^{\prime}(t)[f(t)-f(b)] d t .
\end{aligned}
$$

If $g$ is a function which maps an interval $I$ of the real line to the real numbers, and is both continuous and injective then we can define the g-mean of two numbers $a, b \in I$ as

$$
M_{g}(a, b):=g^{-1}\left(\frac{g(a)+g(b)}{2}\right) .
$$


If $I=\mathbb{R}$ and $g(t)=t$ is the identity function, then $M_{g}(a, b)=A(a, b):=\frac{a+b}{2}$, the arithmetic mean. If $I=(0, \infty)$ and $g(t)=\ln t$, then $M_{g}(a, b)=G(a, b):=\sqrt{a b}$, the geometric mean. If $I=(0, \infty)$ and $g(t)=\frac{1}{t}$, then $M_{g}(a, b)=H(a, b):=\frac{2 a b}{a+b}$, the harmonic mean. If $I=(0, \infty)$ and $g(t)=t^{p}, p \neq 0$, then $M_{g}(a, b)=M_{p}(a, b):=\left(\frac{a^{p}+b^{p}}{2}\right)^{1 / p}$, the power mean with exponent $p$. Finally, if $I=\mathbb{R}$ and $g(t)=\exp t$, then

$$
M_{g}(a, b)=\operatorname{LME}(a, b):=\ln \left(\frac{\exp a+\exp b}{2}\right),
$$

the LogMeanExp function.

Using the $g$-mean of two numbers we can introduce

$$
\begin{aligned}
P_{k, g, a+, b-} f & :=S_{k, g, a+, b-} f\left(M_{g}(a, b)\right) \\
& =\frac{1}{2} \int_{a}^{M_{g}(a, b)} k\left(\frac{g(a)+g(b)}{2}-g(t)\right) g^{\prime}(t) f(t) d t \\
& +\frac{1}{2} \int_{M_{g}(a, b)}^{b} k\left(g(t)-\frac{g(a)+g(b)}{2}\right) g^{\prime}(t) f(t) d t .
\end{aligned}
$$

Using (2.4) and (2.5) we have the representations

$$
\begin{aligned}
& P_{k, g, a+, b-} f \\
& =K\left(\frac{g(b)-g(a)}{2}\right) f\left(M_{g}(a, b)\right) \\
& +\frac{1}{2} \int_{a}^{M_{g}(a, b)} k\left(\frac{g(a)+g(b)}{2}-g(t)\right) g^{\prime}(t)\left[f(t)-f\left(M_{g}(a, b)\right)\right] d t \\
& +\frac{1}{2} \int_{M_{g}(a, b)}^{b} k\left(g(t)-\frac{g(a)+g(b)}{2}\right) g^{\prime}(t)\left[f(t)-f\left(M_{g}(a, b)\right)\right] d t
\end{aligned}
$$

and

$$
\begin{aligned}
& P_{k, g, a+, b-f} \\
& =K\left(\frac{g(b)-g(a)}{2}\right) \frac{f(b)+f(a)}{2} \\
& +\frac{1}{2} \int_{a}^{M_{g}(a, b)} k\left(\frac{g(a)+g(b)}{2}-g(t)\right) g^{\prime}(t)[f(t)-f(a)] d t \\
& +\frac{1}{2} \int_{M_{g}(a, b)}^{b} k\left(g(t)-\frac{g(a)+g(b)}{2}\right) g^{\prime}(t)[f(t)-f(b)] d t .
\end{aligned}
$$

\section{Some identities for the dual operator $\breve{S}_{k, g, a+, b-}$}

Observe that

$$
S_{k, g, x+} f(b)=\int_{x}^{b} k(g(b)-g(t)) g^{\prime}(t) f(t) d t, x \in[a, b)
$$

and

$$
S_{k, g, x-} f(a)=\int_{a}^{x} k(g(t)-g(a)) g^{\prime}(t) f(t) d t, x \in(a, b] .
$$

Define also the mixed operator

$$
\begin{aligned}
& \breve{S}_{k, g, a+, b-} f(x) \\
& :=\frac{1}{2}\left[S_{k, g, x+} f(b)+S_{k, g, x-} f(a)\right] \\
& =\frac{1}{2}\left[\int_{x}^{b} k(g(b)-g(t)) g^{\prime}(t) f(t) d t+\int_{a}^{x} k(g(t)-g(a)) g^{\prime}(t) f(t) d t\right]
\end{aligned}
$$

for any $x \in(a, b)$. 
Lemma 3.1. With the above assumptions for $k, g$ and $f$ we have

$$
\begin{aligned}
\breve{S}_{k, g, a+, b-} f(x) & =\frac{1}{2}[\lambda K(g(b)-g(x))+\gamma K(g(x)-g(a))] \\
& +\frac{1}{2} \int_{a}^{x} k(g(t)-g(a)) g^{\prime}(t)[f(t)-\gamma] d t \\
& +\frac{1}{2} \int_{x}^{b} k(g(b)-g(t)) g^{\prime}(t)[f(t)-\lambda] d t
\end{aligned}
$$

for any $\lambda, \gamma \in \mathbb{C}$.

Proof. We have, by taking the derivative over $t$ and using the chain rule, that

$$
[K(g(b)-g(t))]^{\prime}=K^{\prime}(g(b)-g(t))(g(b)-g(t))^{\prime}=-k(g(b)-g(t)) g^{\prime}(t)
$$

for $t \in(x, b)$ and

$$
[K(g(t)-g(a))]^{\prime}=K^{\prime}(g(t)-g(a))(g(t)-g(a))^{\prime}=k(g(t)-g(a)) g^{\prime}(t)
$$

for $t \in(a, x)$.

For any $\lambda, \gamma \in \mathbb{C}$ we have

$$
\begin{aligned}
& \int_{x}^{b} k(g(b)-g(t)) g^{\prime}(t)[f(t)-\lambda] d t \\
& =\int_{x}^{b} k(g(b)-g(t)) g^{\prime}(t) f(t) d t-\lambda \int_{x}^{b} k(g(b)-g(t)) g^{\prime}(t) d t \\
& =S_{k, g, x+} f(b)+\lambda \int_{x}^{b}[K(g(b)-g(t))]^{\prime} d t \\
& =S_{k, g, x+} f(b)-\lambda K(g(b)-g(x))
\end{aligned}
$$

and

$$
\begin{aligned}
& \int_{a}^{x} k(g(t)-g(a)) g^{\prime}(t)[f(t)-\gamma] d t \\
& =\int_{a}^{x} k(g(t)-g(a)) g^{\prime}(t) f(t) d t-\gamma \int_{a}^{x} k(g(t)-g(a)) g^{\prime}(t) d t \\
& =\int_{a}^{x} k(g(t)-g(a)) g^{\prime}(t) f(t) d t-\gamma \int_{a}^{x}[K(g(t)-g(a))]^{\prime} d t \\
& =\int_{a}^{x} k(g(t)-g(a)) g^{\prime}(t) f(t) d t-\gamma K(g(x)-g(a))
\end{aligned}
$$

for $x \in(a, b)$.

If we add the equalities (3.2) and (3.3) and divide by 2 then we get the desired result (3.1).

Corollary 3.2. With the assumptions of Lemma 3.1 we have the Ostrowski type identity

$$
\begin{aligned}
\breve{S}_{k, g, a+, b-} f(x) & =\frac{1}{2}[K(g(b)-g(x))+K(g(x)-g(a))] f(x) \\
& +\frac{1}{2} \int_{a}^{x} k(g(t)-g(a)) g^{\prime}(t)[f(t)-f(x)] d t \\
& +\frac{1}{2} \int_{x}^{b} k(g(b)-g(t)) g^{\prime}(t)[f(t)-f(x)] d t
\end{aligned}
$$

and the trapezoid identity

$$
\begin{aligned}
\breve{S}_{k, g, a+, b-} f(x) & =\frac{1}{2}[K(g(b)-g(x)) f(b)+K(g(x)-g(a)) f(a)] \\
& +\frac{1}{2} \int_{a}^{x} k(g(t)-g(a)) g^{\prime}(t)[f(t)-f(a)] d t \\
& +\frac{1}{2} \int_{x}^{b} k(g(b)-g(t)) g^{\prime}(t)[f(t)-f(b)] d t
\end{aligned}
$$

for $x \in(a, b)$. 
For $x=\frac{a+b}{2}$ we can consider

$$
\begin{aligned}
\breve{M}_{k, g, a+, b-} f & :=\breve{S}_{k, g, a+, b-} f\left(\frac{a+b}{2}\right) \\
& =\frac{1}{2} \int_{\frac{a+b}{2}}^{b} k(g(b)-g(t)) g^{\prime}(t) f(t) d t \\
& +\frac{1}{2} \int_{a}^{\frac{a+b}{2}} k(g(t)-g(a)) g^{\prime}(t) f(t) d t .
\end{aligned}
$$

Using the equalities (3.4) and (3.5), we have

$$
\begin{aligned}
& \breve{M}_{k, g, a+, b-} f \\
& =\frac{1}{2}\left[K\left(g(b)-g\left(\frac{a+b}{2}\right)\right)+K\left(g\left(\frac{a+b}{2}\right)-g(a)\right)\right] f\left(\frac{a+b}{2}\right) \\
& +\frac{1}{2} \int_{a}^{\frac{a+b}{2}} k(g(t)-g(a)) g^{\prime}(t)\left[f(t)-f\left(\frac{a+b}{2}\right)\right] d t \\
& +\frac{1}{2} \int_{\frac{a+b}{2}}^{b} k(g(b)-g(t)) g^{\prime}(t)\left[f(t)-f\left(\frac{a+b}{2}\right)\right] d t
\end{aligned}
$$

and

$$
\begin{aligned}
& \breve{M}_{k, g, a+, b-} f \\
& =\frac{1}{2}\left[K\left(g(b)-g\left(\frac{a+b}{2}\right)\right) f(b)+K\left(g\left(\frac{a+b}{2}\right)-g(a)\right) f(a)\right] \\
& +\frac{1}{2} \int_{a}^{\frac{a+b}{2}} k(g(t)-g(a)) g^{\prime}(t)[f(t)-f(a)] d t \\
& +\frac{1}{2} \int_{\frac{a+b}{2}}^{b} k(g(b)-g(t)) g^{\prime}(t)[f(t)-f(b)] d t .
\end{aligned}
$$

Using the $g$-mean of two numbers we can introduce

$$
\begin{aligned}
\breve{P}_{k, g, a+, b-} f & :=\breve{S}_{k, g, a+, b-} f\left(M_{g}(a, b)\right) \\
& =\frac{1}{2} \int_{M_{g}(a, b)}^{b} k(g(b)-g(t)) g^{\prime}(t) f(t) d t \\
& +\frac{1}{2} \int_{a}^{M_{g}(a, b)} k(g(t)-g(a)) g^{\prime}(t) f(t) d t .
\end{aligned}
$$

Using the equalities (3.4) and (3.5), we have

$$
\begin{aligned}
\breve{P}_{k, g, a+, b-} f & =K\left(\frac{g(b)-g(a)}{2}\right) f\left(M_{g}(a, b)\right) \\
& +\frac{1}{2} \int_{a}^{M_{g}(a, b)} k(g(t)-g(a)) g^{\prime}(t)\left[f(t)-f\left(M_{g}(a, b)\right)\right] d t \\
& +\frac{1}{2} \int_{M_{g}(a, b)}^{b} k(g(b)-g(t)) g^{\prime}(t)\left[f(t)-f\left(M_{g}(a, b)\right)\right] d t
\end{aligned}
$$

and

$$
\begin{aligned}
\breve{P}_{k, g, a+, b-} f & =K\left(\frac{g(b)-g(a)}{2}\right) \frac{f(b)+f(a)}{2} \\
& +\frac{1}{2} \int_{a}^{M_{g}(a, b)} k(g(t)-g(a)) g^{\prime}(t)[f(t)-f(a)] d t \\
& +\frac{1}{2} \int_{M_{g}(a, b)}^{b} k(g(b)-g(t)) g^{\prime}(t)[f(t)-f(b)] d t .
\end{aligned}
$$




\section{Trapezoid functional $T_{k, g, a+, b-}$}

We can also introduce the functional

$$
\begin{aligned}
T_{k, g, a+, b-} f & :=\frac{1}{2}\left[S_{k, g, a+} f(b)+S_{k, g, b-} f(a)\right] \\
& =\frac{1}{2} \int_{a}^{b}[k(g(b)-g(t))+k(g(t)-g(a))] g^{\prime}(t) f(t) d t .
\end{aligned}
$$

We have:

Lemma 4.1. With the assumption of Lemma 2.1, we have

$$
\begin{aligned}
T_{k, g, a+, b-} f & =K(g(b)-g(a)) \delta \\
& +\frac{1}{2} \int_{a}^{b}[k(g(b)-g(t))+k(g(t)-g(a))] g^{\prime}(t)[f(t)-\delta] d t
\end{aligned}
$$

for any $\delta \in \mathbb{C}$.

Proof. Observe that

$$
\begin{aligned}
& \int_{a}^{b}[k(g(b)-g(t))+k(g(t)-g(a))] g^{\prime}(t) d t \\
& =\int_{a}^{b} k(g(b)-g(t)) g^{\prime}(t) d t+\int_{a}^{b} k(g(t)-g(a)) g^{\prime}(t) d t \\
& =-\int_{a}^{b}[K(g(b)-g(t))]^{\prime} d t+\int_{a}^{b}[K(g(t)-g(a))]^{\prime} d t \\
& =-\left.K(g(b)-g(t))\right|_{a} ^{b}+\left.K(g(t)-g(a))\right|_{a} ^{b} \\
& =K(g(b)-g(a))+K(g(b)-g(a))=2 K(g(b)-g(a)) .
\end{aligned}
$$

Therefore

$$
\begin{aligned}
& \frac{1}{2} \int_{a}^{b}[k(g(b)-g(t))+k(g(t)-g(a))] g^{\prime}(t)[f(t)-\delta] d t \\
& =\frac{1}{2} \int_{a}^{b}[k(g(b)-g(t))+k(g(t)-g(a))] g^{\prime}(t) f(t) d t \\
& -\frac{1}{2} \delta \int_{a}^{b}[k(g(b)-g(t))+k(g(t)-g(a))] g^{\prime}(t) d t \\
& =T_{k, g, a+, b-} f-\delta K(g(b)-g(a)),
\end{aligned}
$$

which proves the desired equality (4.1).

Corollary 4.2. With the assumptions of Lemma 4.1 we have the Ostrowski type identity

$$
\begin{aligned}
& T_{k, g, a+, b-} f \\
& =K(g(b)-g(a)) f(x) \\
& +\frac{1}{2} \int_{a}^{b}[k(g(b)-g(t))+k(g(t)-g(a))] g^{\prime}(t)[f(t)-f(x)] d t
\end{aligned}
$$

for any $x \in[a, b]$ and the trapezoid identity

$$
\begin{aligned}
& T_{k, g, a+, b-} f \\
& =K(g(b)-g(a)) \frac{f(a)+f(b)}{2} \\
& +\frac{1}{2} \int_{a}^{b}[k(g(b)-g(t))+k(g(t)-g(a))] g^{\prime}(t)\left[f(t)-\frac{f(a)+f(b)}{2}\right] d t .
\end{aligned}
$$


We observe that for $x=\frac{a+b}{2}$ we obtain from (4.2) that

$$
\begin{aligned}
& T_{k, g, a+, b-} f \\
& =K(g(b)-g(a)) f\left(\frac{a+b}{2}\right) \\
& +\frac{1}{2} \int_{a}^{b}[k(g(b)-g(t))+k(g(t)-g(a))] g^{\prime}(t)\left[f(t)-f\left(\frac{a+b}{2}\right)\right] d t .
\end{aligned}
$$

\section{Inequalities for functions of bounded variation}

We considered the cumulative function $K:[0, \infty) \rightarrow \mathbb{C}$ by

$$
K(t):=\left\{\begin{array}{l}
\int_{0}^{t} k(s) d s \text { if } 0<t \\
0 \text { if } t=0
\end{array}\right.
$$

We also define the function $\mathbf{K}:[0, \infty) \rightarrow[0, \infty)$ by

$$
\mathbf{K}(t):=\left\{\begin{array}{l}
\int_{0}^{t}|k(s)| d s \text { if } 0<t \\
0 \text { if } t=0 .
\end{array}\right.
$$

We observe that if $k$ takes nonnegative values on $(0, \infty)$, as it does in some of the examples in Introduction, then $\mathbf{K}(t)=K(t)$ for $t \in[0, \infty)$.

Theorem 5.1. Assume that the kernel $k$ is defined either on $(0, \infty)$ or on $[0, \infty)$ with complex values and integrable on any finite subinterval. Let $f:[a, b] \rightarrow \mathbb{C}$ be a function of bounded variation on $[a, b]$ and $g$ be a strictly increasing function on $(a, b)$, having a continuous derivative $g^{\prime}$ on $(a, b)$. Then we have the Ostrowski type inequality

$$
\begin{aligned}
& \left|S_{k, g, a+, b-} f(x)-\frac{1}{2}[K(g(b)-g(x))+K(g(x)-g(a))] f(x)\right| \\
& \leq \frac{1}{2}\left[\int_{x}^{b}|k(g(t)-g(x))| \bigvee_{x}^{t}(f) g^{\prime}(t) d t+\int_{a}^{x}|k(g(x)-g(t))| \bigvee_{t}^{x}(f) g^{\prime}(t) d t\right] \\
& \leq \frac{1}{2}\left[\mathbf{K}(g(b)-g(x)) \bigvee_{x}^{b}(f)+\mathbf{K}(g(x)-g(a)) \bigvee_{a}^{x}(f)\right] \\
& \leq \frac{1}{2}\left\{\begin{array}{l}
\max \{\mathbf{K}(g(b)-g(x)), \mathbf{K}(g(x)-g(a))\} \bigvee_{a}^{b}(f) ; \\
{\left[\mathbf{K}^{p}(g(b)-g(x))+\mathbf{K}^{p}(g(x)-g(a))\right]^{1 / p}\left(\left(\bigvee_{a}^{x}(f)\right)^{q}+\left(\bigvee_{x}^{b}(f)\right)^{q}\right)^{1 / q}} \\
\text { with } p, q>1, \frac{1}{p}+\frac{1}{q}=1 ; \\
{[\mathbf{K}(g(b)-g(x))+\mathbf{K}(g(x)-g(a))]\left[\frac{1}{2} \bigvee_{a}^{b}(f)+\frac{1}{2}\left|\bigvee_{a}^{x}(f)-\bigvee_{x}^{b}(f)\right|\right]}
\end{array}\right.
\end{aligned}
$$


and the trapezoid type inequality

$$
\begin{aligned}
& \left|S_{k, g, a+, b-} f(x)-\frac{1}{2}[K(g(b)-g(x)) f(b)+K(g(x)-g(a)) f(a)]\right| \\
& \leq \frac{1}{2}\left[\int_{a}^{x}|k(g(x)-g(t))| \bigvee_{a}^{t}(f) g^{\prime}(t) d t+\int_{x}^{b}|k(g(t)-g(x))| \bigvee_{t}^{b}(f) g^{\prime}(t) d t\right] \\
& \leq \frac{1}{2}\left[\mathbf{K}(g(b)-g(x)) \bigvee_{x}^{b}(f)+\mathbf{K}(g(x)-g(a)) \bigvee_{a}^{x}(f)\right] \\
& \left\{\begin{array}{l}
\max \{\mathbf{K}(g(b)-g(x)), \mathbf{K}(g(x)-g(a))\} \bigvee_{a}^{b}(f) ; \\
{\left[\mathbf{K}^{p}(g(b)-g(x))+\mathbf{K}^{p}(g(x)-g(a))\right]^{1 / p}}
\end{array}\right. \\
& \leq \frac{1}{2}\left\{\begin{array}{l}
{\left[\mathbf{K}^{p}(g(b)-g(x))+\mathbf{K}^{p}(g(x)-g(a))\right]^{1 / p}} \\
\times\left(\left(\bigvee_{a}^{x}(f)\right)^{q}+\left(\bigvee_{x}^{b}(f)\right)^{q}\right)^{1 / q} \\
\text { with } p, q>1, \frac{1}{p}+\frac{1}{q}=1 \\
\\
{[\mathbf{K}(g(b)-g(x))+\mathbf{K}(g(x)-g(a))]} \\
\times\left[\frac{1}{2} \bigvee_{a}^{b}(f)+\frac{1}{2}\left|\bigvee_{a}^{x}(f)-\bigvee_{x}^{b}(f)\right|\right]
\end{array}\right.
\end{aligned}
$$

for any $x \in(a, b)$.

Proof. Using the equality (2.4) we have

$$
\begin{aligned}
& \left|S_{k, g, a+, b-} f(x)-\frac{1}{2}[K(g(b)-g(x))+K(g(x)-g(a))] f(x)\right| \\
& \leq \frac{1}{2}\left|\int_{a}^{x} k(g(x)-g(t)) g^{\prime}(t)[f(t)-f(x)] d t\right| \\
& +\frac{1}{2}\left|\int_{x}^{b} k(g(t)-g(x)) g^{\prime}(t)[f(t)-f(x)] d t\right| \\
& \leq \frac{1}{2} \int_{a}^{x}\left|k(g(x)-g(t)) g^{\prime}(t)[f(t)-f(x)]\right| d t \\
& +\frac{1}{2} \int_{x}^{b}\left|k(g(t)-g(x)) g^{\prime}(t)[f(t)-f(x)]\right| d t \\
& =\frac{1}{2} \int_{a}^{x}|k(g(x)-g(t))||f(x)-f(t)| g^{\prime}(t) d t \\
& +\frac{1}{2} \int_{x}^{b}|k(g(t)-g(x))||f(t)-f(x)| g^{\prime}(t) d t \\
& =: B(x)
\end{aligned}
$$

for $x \in(a, b)$.

Since $f$ is of bounded variation, then

$$
|f(x)-f(t)| \leq \bigvee_{t}^{x}(f) \leq \bigvee_{a}^{x}(f) \text { for } a<t \leq x \leq b
$$

and

$$
|f(t)-f(x)| \leq \bigvee_{x}^{t}(f) \leq \bigvee_{x}^{b}(f) \text { for } a \leq x \leq t<b
$$


Therefore

$$
\begin{aligned}
B(x) & \leq \frac{1}{2} \int_{a}^{x}|k(g(x)-g(t))| \bigvee_{t}^{x}(f) g^{\prime}(t) d t \\
& +\frac{1}{2} \int_{x}^{b}|k(g(t)-g(x))| \bigvee_{x}^{t}(f) g^{\prime}(t) d t \\
& \leq \frac{1}{2} \bigvee_{a}^{x}(f) \int_{a}^{x}|k(g(x)-g(t))| g^{\prime}(t) d t \\
& +\frac{1}{2} \bigvee_{x}^{b}(f) \int_{x}^{b}|k(g(t)-g(x))| g^{\prime}(t) d t \\
& =: C(x)
\end{aligned}
$$

for $x \in(a, b)$.

We have, by taking the derivative over $t$ and using the chain rule, that

$$
[\mathbf{K}(g(x)-g(t))]^{\prime}=\mathbf{K}^{\prime}(g(x)-g(t))(g(x)-g(t))^{\prime}=-|k(g(x)-g(t))| g^{\prime}(t)
$$

for $t \in(a, x)$ and

$$
[\mathbf{K}(g(t)-g(x))]^{\prime}=\mathbf{K}^{\prime}(g(t)-g(x))(g(t)-g(x))^{\prime}=|k(g(t)-g(x))| g^{\prime}(t)
$$

for $t \in(x, b)$.

Then

$$
\int_{a}^{x}|k(g(x)-g(t))| g^{\prime}(t) d t=-\int_{a}^{x}[\mathbf{K}(g(x)-g(t))]^{\prime} d t=\mathbf{K}(g(x)-g(a))
$$

and

$$
\int_{x}^{b}|k(g(t)-g(x))| g^{\prime}(t) d t=\int_{x}^{b}[\mathbf{K}(g(t)-g(x))]^{\prime} d t=\mathbf{K}(g(b)-g(x))
$$

giving that

$$
C(x)=\frac{1}{2}\left[\mathbf{K}(g(b)-g(x)) \bigvee_{x}^{b}(f)+\mathbf{K}(g(x)-g(a)) \bigvee_{a}^{x}(f)\right],
$$

for $x \in(a, b)$, which proves the first and the second inequality in (5.1).

The last part of (4.1 is obvious by making use of the elementary Hölder type inequalities for positive real numbers $c, d, m$, $n \geq 0$

$$
m c+n d \leq\left\{\begin{array}{l}
\max \{m, n\}(c+d) \\
\left(m^{p}+n^{p}\right)^{1 / p}\left(c^{q}+d^{q}\right)^{1 / q} \text { with } p, q>1, \frac{1}{p}+\frac{1}{q}=1 .
\end{array}\right.
$$


Further, by the identity (2.5) we have, as above,

$$
\begin{aligned}
& \left|S_{k, g, a+, b-} f(x)-\frac{1}{2}[K(g(b)-g(x)) f(b)+K(g(x)-g(a)) f(a)]\right| \\
& \leq \frac{1}{2} \int_{a}^{x}|k(g(x)-g(t))||f(t)-f(a)| g^{\prime}(t) d t \\
& +\frac{1}{2} \int_{x}^{b}|k(g(t)-g(x))||f(t)-f(b)| g^{\prime}(t) d t \\
& \leq \frac{1}{2} \int_{a}^{x}|k(g(x)-g(t))| \bigvee_{a}^{t}(f) g^{\prime}(t) d t \\
& +\frac{1}{2} \int_{x}^{b}|k(g(t)-g(x))| \bigvee_{t}^{b}(f) g^{\prime}(t) d t \\
& \leq \frac{1}{2} \bigvee_{a}^{x}(f) \int_{a}^{x}|k(g(x)-g(t))| g^{\prime}(t) d t \\
& +\frac{1}{2} \bigvee_{x}^{b}(f) \int_{x}^{b}|k(g(t)-g(x))| g^{\prime}(t) d t \\
& =\frac{1}{2} \mathbf{K}(g(x)-g(a)) \bigvee_{a}^{x}(f)+\frac{1}{2} \mathbf{K}(g(b)-g(x)) \bigvee_{x}^{b}(f),
\end{aligned}
$$

which proves (5.2).

The following particular case for the functional

$$
\begin{aligned}
P_{k, g, a+, b-} f & :=S_{k, g, a+, b-} f\left(M_{g}(a, b)\right) \\
& =\frac{1}{2} \int_{a}^{M_{g}(a, b)} k\left(\frac{g(b)+g(a)}{2}-g(t)\right) g^{\prime}(t) f(t) d t \\
& +\frac{1}{2} \int_{M_{g}(a, b)}^{b} k\left(g(t)-\frac{g(b)+g(a)}{2}\right) g^{\prime}(t) f(t) d t .
\end{aligned}
$$

is of interest:

Corollary 5.2. With the assumptions of Theorem 5.1 we have

$$
\begin{aligned}
\left|P_{k, g, a+, b-} f-K\left(\frac{g(b)-g(a)}{2}\right) f\left(M_{g}(a, b)\right)\right| & \leq \frac{1}{2} \int_{M_{g}(a, b)}^{b}\left|k\left(g(t)-\frac{g(b)+g(a)}{2}\right)\right| \bigvee_{M_{g}(a, b)}^{t}(f) g^{\prime}(t) d t \\
& +\frac{1}{2} \int_{a}^{M_{g}(a, b)} \mid k\left(\frac{g(b)+g(a)}{2}-g(t)\right) \bigvee_{t}^{M_{g}(a, b)}(f) g^{\prime}(t) d t \\
& \leq \frac{1}{2} \mathbf{K}\left(\frac{g(b)-g(a)}{2}\right) \bigvee_{b}^{b}(f)
\end{aligned}
$$

and

$$
\begin{aligned}
\left|P_{k, g, a+, b-} f-K\left(\frac{g(b)-g(a)}{2}\right) \frac{f(b)+f(a)}{2}\right| & \leq \frac{1}{2} \int_{a}^{M_{g}(a, b)}\left|k\left(\frac{g(b)+g(a)}{2}-g(t)\right)\right| \bigvee_{a}^{t}(f) g^{\prime}(t) d t \\
& +\frac{1}{2} \int_{M_{g}(a, b)}^{b}\left|k\left(g(t)-\frac{g(b)+g(a)}{2}\right)\right| \bigvee_{t}^{b}(f) g^{\prime}(t) d t \\
& \leq \frac{1}{2} \mathbf{K}\left(\frac{g(b)-g(a)}{2}\right) \bigvee_{b}^{b}(f) .
\end{aligned}
$$

We have: 
Theorem 5.3. With the assumptions of Theorem 5.1 we have the Ostrowski type inequality

$$
\begin{aligned}
& \left|\breve{S}_{k, g, a+, b-} f(x)-\frac{1}{2}[K(g(b)-g(x))+K(g(x)-g(a))] f(x)\right| \\
& \leq \frac{1}{2} \int_{a}^{x}|k(g(t)-g(a))| \bigvee_{t}^{x}(f) g^{\prime}(t) d t+\frac{1}{2} \int_{x}^{b}|k(g(b)-g(t))| \bigvee_{x}^{t}(f) g^{\prime}(t) d t \\
& \leq \frac{1}{2}\left[\mathbf{K}(g(b)-g(x)) \bigvee_{x}^{b}(f)+\mathbf{K}(g(x)-g(a)) \bigvee_{a}^{x}(f)\right] \\
& \leq \frac{1}{2}\left\{\begin{array}{l}
{\left[\mathbf{K} p(g(b)-g(x))+\mathbf{K}^{p}(g(x)-g(a))\right]^{1 / p}\left(\left(\bigvee_{a}^{x}(f)\right)^{q}+\left(\bigvee_{x}^{b}(f)\right)^{q}\right)^{1 / q}} \\
w i t h p, q>1, \frac{1}{p}+\frac{1}{q}=1 ; \\
{[\mathbf{K}(g(b)-g(x))+\mathbf{K}(g(x)-g(a))]\left[\frac{1}{2} \bigvee_{a}^{b}(f)+\frac{1}{2}\left|\bigvee_{a}^{x}(f)-\bigvee_{x}^{b}(f)\right|\right]}
\end{array}\right.
\end{aligned}
$$

and the trapezoid inequality

$$
\begin{aligned}
& \left|\breve{S}_{k, g, a+, b-} f(x)-\frac{1}{2}[K(g(b)-g(x)) f(b)+K(g(x)-g(a)) f(a)]\right| \\
& \leq \frac{1}{2} \int_{a}^{x}|k(g(t)-g(a))| \bigvee_{a}^{t}(f) g^{\prime}(t) d t+\frac{1}{2} \int_{x}^{b}|k(g(b)-g(t))| \bigvee_{t}^{b}(f) g^{\prime}(t) d t \\
& \leq \frac{1}{2}\left[\mathbf{K}(g(b)-g(x)) \bigvee_{x}^{b}(f)+\mathbf{K}(g(x)-g(a)) \bigvee_{a}^{x}(f)\right] \\
& \leq \frac{1}{2}\left\{\begin{array}{l}
\max \{\mathbf{K}(g(b)-g(x)), \mathbf{K}(g(x)-g(a))\} \bigvee_{a}^{b}(f) ; \\
w i t h p, q>1, \frac{1}{p}+\frac{1}{q}=1 ; \\
{[\mathbf{K}(g(b)-g(x))+\mathbf{K}(g(x)-g(a))]\left[\frac{1}{2} \bigvee_{a}^{b}(f)+\frac{1}{2}\left|\bigvee_{a}^{x}(f)-\bigvee_{x}^{b}(f)\right|\right]}
\end{array}\right.
\end{aligned}
$$

for any $x \in(a, b)$. 
Proof. Using the identity (3.4) we have

$$
\begin{aligned}
& \left|\breve{S}_{k, g, a+, b-} f(x)-\frac{1}{2}[K(g(b)-g(x))+K(g(x)-g(a))] f(x)\right| \\
& \leq \frac{1}{2} \int_{a}^{x}|k(g(t)-g(a))||f(t)-f(x)| g^{\prime}(t) d t \\
& +\frac{1}{2} \int_{x}^{b}|k(g(b)-g(t))||f(t)-f(x)| g^{\prime}(t) d t \\
& \leq \frac{1}{2} \int_{a}^{x}|k(g(t)-g(a))| \bigvee_{t}^{x}(f) g^{\prime}(t) d t \\
& +\frac{1}{2} \int_{x}^{b}|k(g(b)-g(t))| \bigvee_{x}^{t}(f) g^{\prime}(t) d t \\
& \leq \frac{1}{2} \bigvee_{a}^{x}(f) \int_{a}^{x}|k(g(t)-g(a))| g^{\prime}(t) d t \\
& \left.+\frac{1}{2} \bigvee_{x}^{b}(f) \int_{x}^{b}|k(g(b)-g(t))| g^{\prime}(t) d t \quad{ }_{x}^{x} \underset{a}{b}(f)+\mathbf{K}(g(b)-g(x)) \bigvee_{x}(f)\right] \\
& =\frac{1}{2}[\mathbf{K}(g(x)-g(a)]
\end{aligned}
$$

for any $x \in(a, b)$, which proves (5.5).

By the identity (3.5) we have

$$
\begin{aligned}
& \left|\breve{S}_{k, g, a+, b-} f(x)-\frac{1}{2}[K(g(b)-g(x)) f(b)+K(g(x)-g(a)) f(a)]\right| \\
& \leq \frac{1}{2} \int_{a}^{x}|k(g(t)-g(a))||f(t)-f(a)| g^{\prime}(t) d t \\
& +\frac{1}{2} \int_{x}^{b}|k(g(b)-g(t))||f(b)-f(t)| g^{\prime}(t) d t \\
& \leq \frac{1}{2} \int_{a}^{x}|k(g(t)-g(a))| \bigvee_{a}^{t}(f) g^{\prime}(t) d t \\
& +\frac{1}{2} \int_{x}^{b}|k(g(b)-g(t))| \bigvee_{t}^{b}(f) g^{\prime}(t) d t \\
& \leq \frac{1}{2} \bigvee_{a}^{x}(f) \int_{a}^{x}|k(g(t)-g(a))| g^{\prime}(t) d t \\
& +\frac{1}{2} \bigvee_{x}^{b}(f) \int_{x}^{b}|k(g(b)-g(t))| g^{\prime}(t) d t \\
& =\frac{1}{2}\left[\mathbf{K}(g(x)-g(a)) \bigvee_{a}^{x}(f)+\mathbf{K}(g(b)-g(x)) \bigvee_{x}^{b}(f)\right]
\end{aligned}
$$

for any $x \in(a, b)$, which proves (5.6).

Also, we have the particular inequalities for

$$
\begin{aligned}
\breve{P}_{k, g, a+, b-} f & :=\breve{S}_{k, g, a+, b-} f\left(M_{g}(a, b)\right) \\
& =\frac{1}{2} \int_{M_{g}(a, b)}^{b} k(g(b)-g(t)) g^{\prime}(t) f(t) d t \\
& +\frac{1}{2} \int_{a}^{M_{g}(a, b)} k(g(t)-g(a)) g^{\prime}(t) f(t) d t .
\end{aligned}
$$


Corollary 5.4. With the assumptions of Theorem 5.1 we have

$$
\begin{aligned}
\left|\breve{P}_{k, g, a+, b-} f-K\left(\frac{g(b)-g(a)}{2}\right) \frac{f(b)+f(a)}{2}\right| & \leq \frac{1}{2} \int_{a}^{M_{g}(a, b)}|k(g(t)-g(a))| \bigvee_{t}^{M_{g}(a, b)}(f) g^{\prime}(t) d t \\
& +\frac{1}{2} \int_{M_{g}(a, b)}^{b}|k(g(b)-g(t))| \bigvee_{M_{g}(a, b)}^{t}(f) g^{\prime}(t) d t \\
& \leq \frac{1}{2} \mathbf{K}\left(\frac{g(b)-g(a)}{2}\right) \bigvee_{b}^{b}(f)
\end{aligned}
$$

and

$$
\begin{aligned}
\left|\breve{P}_{k, g, a+, b-} f-K\left(\frac{g(b)-g(a)}{2}\right) \frac{f(b)+f(a)}{2}\right| & \leq \frac{1}{2} \int_{a}^{M_{g}(a, b)}|k(g(t)-g(a))| \bigvee_{a}^{t}(f) g^{\prime}(t) d t \\
& +\frac{1}{2} \int_{M_{g}(a, b)}^{b}|k(g(b)-g(t))| \bigvee_{t}^{b}(f) g^{\prime}(t) d t \\
& \leq \frac{1}{2} \mathbf{K}\left(\frac{g(b)-g(a)}{2}\right) \bigvee_{b}^{b}(f) .
\end{aligned}
$$

Finally, we have the following result for the trapezoid functional

$$
\begin{aligned}
T_{k, g, a+, b-} f & :=\frac{1}{2}\left[S_{k, g, a+} f(b)+S_{k, g, b-} f(a)\right] \\
& =\frac{1}{2} \int_{a}^{b}[k(g(b)-g(t))+k(g(t)-g(a))] g^{\prime}(t) f(t) d t .
\end{aligned}
$$

Theorem 5.5. With the assumptions of Theorem 5.1 we have the trapezoid type inequality

$$
\left|T_{k, g, a+, b-} f-K(g(b)-g(a)) \frac{f(a)+f(b)}{2}\right| \leq \frac{1}{2} \mathbf{K}(g(b)-g(a)) \bigvee_{a}^{b}(f) .
$$

Proof. From the identity (4.3) we have

$$
\begin{aligned}
& \left|T_{k, g, a+, b-} f-K(g(b)-g(a)) \frac{f(a)+f(b)}{2}\right| \\
& \leq \frac{1}{2} \int_{a}^{b}|k(g(b)-g(t))+k(g(t)-g(a))|\left|f(t)-\frac{f(a)+f(b)}{2}\right| g^{\prime}(t) d t \\
& \leq \frac{1}{2} \int_{a}^{b}[|k(g(b)-g(t))|+|k(g(t)-g(a))|]\left|f(t)-\frac{f(a)+f(b)}{2}\right| g^{\prime}(t) d t \\
& =: D .
\end{aligned}
$$

Since $f:[a, b] \rightarrow \mathbb{C}$ is of bounded variation, then for any $t \in[a, b]$ we have

$$
\begin{aligned}
\left|f(t)-\frac{f(a)+f(b)}{2}\right| & =\left|\frac{f(t)-f(a)+f(t)-f(b)}{2}\right| \\
& \leq \frac{1}{2}[|f(t)-f(a)|+|f(b)-f(t)|] \leq \frac{1}{2} \bigvee_{a}^{b}(f) .
\end{aligned}
$$

Therefore

$$
\begin{aligned}
D & \leq \frac{1}{4} \bigvee_{a}^{b}(f) \int_{a}^{b}[|k(g(b)-g(t))|+|k(g(t)-g(a))|] g^{\prime}(t) d t \\
& =\frac{1}{4} \bigvee_{a}^{b}(f)[\mathbf{K}(g(b)-g(a))+\mathbf{K}(g(b)-g(a))]=\frac{1}{2} \mathbf{K}(g(b)-g(a)) \bigvee_{a}^{b}(f),
\end{aligned}
$$

which proves the desired result (5.7). 


\section{Example for an exponential kernel}

The above inequalities may be written for all the particular fractional integrals introduced in the introduction.

If we take, for instance $k(t)=\frac{1}{\Gamma(\alpha)} t^{\alpha-1}$, where $\Gamma$ is the Gamma function, then we recapture the results for the generalized left- and right-sided Riemann-Liouville fractional integrals of a function $f$ with respect to another function $g$ on $[a, b]$ as outlined in [5].

For $\alpha, \beta \in \mathbb{R}$ we consider the kernel $k(t):=\exp [(\alpha+\beta i) t], t \in \mathbb{R}$. We have

$$
K(t)=\frac{\exp [(\alpha+\beta i) t]-1}{(\alpha+\beta i)}, \text { if } t \in \mathbb{R}
$$

for $\alpha, \beta \neq 0$.

Also, we have

$$
|k(s)|:=|\exp [(\alpha+\beta i) s]|=\exp (\alpha s) \text { for } s \in \mathbb{R}
$$

and

$$
\mathbf{K}(t)=\int_{0}^{t} \exp (\alpha s) d s=\frac{\exp (\alpha t)-1}{\alpha} \text { if } 0<t,
$$

for $\alpha \neq 0$.

Let $f:[a, b] \rightarrow \mathbb{C}$ be a function of bounded variation on $[a, b]$ and $g$ be a strictly increasing function on $(a, b)$, having a continuous derivative $g^{\prime}$ on $(a, b)$. We have

$$
\begin{aligned}
\mathscr{E}_{g, a+, b-}^{\alpha+\beta i} f(x) & =\frac{1}{2} \int_{a}^{x} \exp [(\alpha+\beta i)(g(x)-g(t))] g^{\prime}(t) f(t) d t \\
& +\frac{1}{2} \int_{x}^{b} \exp [(\alpha+\beta i)(g(t)-g(x))] g^{\prime}(t) f(t) d t
\end{aligned}
$$

for $x \in(a, b)$.

If $g=\ln h$ where $h:[a, b] \rightarrow(0, \infty)$ is a strictly increasing function on $(a, b)$, having a continuous derivative $h^{\prime}$ on $(a, b)$, then we can consider the following operator as well

$$
\begin{aligned}
& \kappa_{h, a+, b-}^{\alpha+\beta i} f(x) \\
& :=\mathscr{E}_{\ln h, a+, b-}^{\alpha+\beta i} f(x) \\
& =\frac{1}{2}\left[\int_{a}^{x}\left(\frac{h(x)}{h(t)}\right)^{\alpha+\beta i} \frac{h^{\prime}(t)}{h(t)} f(t) d t+\int_{x}^{b}\left(\frac{h(t)}{h(x)}\right)^{\alpha+\beta i} \frac{h^{\prime}(t)}{h(t)} f(t) d t\right]
\end{aligned}
$$

for $x \in(a, b)$.

By using the inequality (5.1) we have for $x \in(a, b)$ that 


$$
\begin{aligned}
& \mid \mathscr{E}_{g, a+, b-}^{\alpha+\beta i} f(x) \\
& -\frac{1}{2}\left[\frac{\exp [(\alpha+\beta i)(g(b)-g(x))]-1+\exp [(\alpha+\beta i)(g(x)-g(a))]-1}{(\alpha+\beta i)}\right] f(x) \\
& \leq \frac{1}{2}\left[\int_{x}^{b} \exp (\alpha(g(t)-g(x))) g^{\prime}(t) \bigvee_{x}^{t}(f) d t+\int_{a}^{x} \exp (\alpha(g(x)-g(t))) g^{\prime}(t) \bigvee_{t}^{x}(f) d t\right] \\
& \leq \frac{1}{2}\left[\frac{\exp (\alpha(g(b)-g(x)))-1}{\alpha} \bigvee_{x}^{b}(f)+\frac{\exp (\alpha(g(x)-g(a)))-1}{\alpha} \bigvee_{a}^{x}(f)\right] \\
& \leq \frac{1}{2}\left\{\begin{array}{l}
\max \left\{\frac{\exp (\alpha(g(b)-g(x)))-1}{\alpha}, \frac{\exp (\alpha(g(x)-g(a)))-1}{\alpha}\right\} \bigvee_{a}^{b}(f) ; \\
{\left[\left(\frac{\exp (\alpha(g(b)-g(x)))-1}{\alpha}\right)^{p}+\left(\frac{\exp (\alpha(g(x)-g(a)))-1}{\alpha}\right)^{p}\right]^{1 / p}\left(\left(\bigvee_{a}^{x}(f)\right)^{q}+\left(\bigvee_{x}^{b}(f)\right)^{q}\right)^{1 / q}} \\
\text { with } p, q>1, \frac{1}{p}+\frac{1}{q}=1 ; \\
{\left[\frac{\exp (\alpha(g(b)-g(x)))-1+\exp (\alpha(g(x)-g(a)))-1}{\alpha}\right]\left[\frac{1}{2} \bigvee_{a}^{b}(f)+\frac{1}{2}\left|\bigvee_{a}^{x}(f)-\bigvee_{x}^{b}(f)\right|\right]}
\end{array}\right.
\end{aligned}
$$

for $\alpha, \beta \in \mathbb{R}$ with $\alpha \neq 0$.

By using the inequality (5.2) we also have for $x \in(a, b)$ that

$$
\begin{aligned}
& \left|\mathscr{E}_{g, a+, b-}^{\alpha+\beta i} f(x)-\frac{1}{2}\left[\frac{(\exp [(\alpha+\beta i)(g(b)-g(x))]-1) f(b)+(\exp [(\alpha+\beta i)(g(x)-g(a))]-1) f(a)}{(\alpha+\beta i)}\right]\right| \\
& \leq \frac{1}{2}\left[\int_{a}^{x} \exp (\alpha(g(t)-g(x))) g^{\prime}(t) \bigvee_{a}^{t}(f) d t+\int_{x}^{b} \exp (\alpha(g(x)-g(t))) g^{\prime}(t) \bigvee_{t}^{b}(f) d t\right] \\
& \leq \frac{1}{2}\left[\frac{\exp (\alpha(g(b)-g(x)))-1}{\alpha} \bigvee_{x}^{b}(f)+\frac{\exp (\alpha(g(x)-g(a)))-1}{\alpha} \bigvee_{a}^{x}(f)\right] \\
& \leq \frac{1}{2}\left\{\begin{array}{l}
\max \left\{\frac{\exp (\alpha(g(b)-g(x)))-1}{\alpha}, \frac{\exp (\alpha(g(x)-g(a)))}{\alpha}\right. \\
{\left[\left(\frac{\exp (\alpha(g(b)-g(x)))-1}{\alpha}\right)^{p}+\left(\frac{\exp (\alpha(g(x)-g(a)))}{\alpha}\right.\right.} \\
\times\left(\left(\bigvee_{a}^{x}(f)\right)^{q}+\left(\bigvee_{x}^{b}(f)\right)^{q}\right)^{1 / q} \\
\text { with } p, q>1, \frac{1}{p}+\frac{1}{q}=1 \\
{\left[\frac{\exp (\alpha(g(b)-g(x)))-1+\exp (\alpha(g(x)-g(a)))-1}{\alpha}\right]} \\
\times\left[\frac{1}{2} \bigvee_{a}^{b}(f)+\frac{1}{2}\left|\bigvee_{a}^{x}(f)-\bigvee_{x}^{b}(f)\right|\right]
\end{array}\right.
\end{aligned}
$$

for $\alpha, \beta \in \mathbb{R}$ with $\alpha \neq 0$.

If we denote

$$
\begin{aligned}
\overline{\mathscr{E}}_{g, a+, b-}^{\alpha+\beta i} f & :=\mathscr{E}_{g, a+, b-}^{\alpha+\beta i} f\left(M_{g}(a, b)\right) \\
& =\frac{1}{2} \int_{a}^{x} \exp \left[(\alpha+\beta i)\left(\frac{g(b)+g(a)}{2}-g(t)\right)\right] g^{\prime}(t) f(t) d t \\
& +\frac{1}{2} \int_{x}^{b} \exp \left[(\alpha+\beta i)\left(g(t)-\frac{g(b)+g(a)}{2}\right)\right] g^{\prime}(t) f(t) d t
\end{aligned}
$$


then by (5.3) and (5.4) we have the simpler results

$$
\begin{aligned}
& \left|\overline{\mathscr{E}}_{g, a+, b-}^{\alpha+\beta i} f-\frac{\exp \left[(\alpha+\beta i) \frac{g(b)-g(a)}{2}\right]-1}{(\alpha+\beta i)} f\left(M_{g}(a, b)\right)\right| \leq \frac{1}{2} \int_{M_{g}(a, b)}^{b} \exp \left(\alpha\left(g(t)-\frac{g(b)+g(a)}{2}\right)\right) g^{\prime}(t) \bigvee_{M_{g}(a, b)}^{t}(f) d t \\
& +\frac{1}{2} \int_{a}^{M_{g}(a, b)} \exp \left(\alpha\left(\frac{g(b)+g(a)}{2}-g(t)\right)\right) g^{\prime}(t) \bigvee_{t}^{M_{g}(a, b)}(f) d t \\
& \leq \frac{1}{2} \frac{\exp \left(\alpha\left(\frac{g(b)-g(a)}{2}\right)\right)-1}{\alpha} \bigvee_{b}^{b}(f)
\end{aligned}
$$

and

$$
\begin{aligned}
& \left|\overline{\mathscr{E}}_{g, a+, b-}^{\alpha+\beta i} f-\frac{\exp \left[(\alpha+\beta i) \frac{g(b)-g(a)}{2}\right]-1}{(\alpha+\beta i)} \frac{f(b)+f(a)}{2}\right| \leq \frac{1}{2} \int_{a}^{M_{g}(a, b)} \exp \left(\alpha\left(g(t)-\frac{g(b)+g(a)}{2}\right)\right) g^{\prime}(t) \bigvee_{a}^{t}(f) d t \\
& +\frac{1}{2} \int_{M_{g}(a, b)}^{b} \exp \left(\alpha\left(\frac{g(b)+g(a)}{2}-g(t)\right)\right) g^{\prime}(t) \bigvee_{t}^{b}(f) d t \\
& \leq \frac{1}{2} \frac{\exp \left(\alpha\left(\frac{g(b)-g(a)}{2}\right)\right)-1}{\alpha} \bigvee_{b}^{b}(f)
\end{aligned}
$$

In particular, if we take in (6.1) and (6.2) $g=\ln t, t \in[a, b] \subset(0, \infty)$, then by using the notation $G(\gamma, \delta):=\sqrt{\gamma \delta}$ for the geometric mean of the positive real numbers $\gamma, \delta>0$ we have

$$
\begin{aligned}
\left|\bar{\kappa}_{a+, b-}^{\alpha+\beta i} f-\frac{\left(\frac{b}{a}\right)^{\alpha+\beta i}-1}{(\alpha+\beta i)} f(G(a, b))\right| & \leq \frac{1}{2} \int_{G(a, b)}^{b}\left(\frac{t}{G(a, b)}\right)^{\alpha} \frac{1}{t} \bigvee_{G(a, b)}^{t}(f) d t+\frac{1}{2} \int_{a}^{G(a, b)}\left(\frac{G(a, b)}{t}\right)^{\alpha} \frac{1}{t} \bigvee_{t}^{G(a, b)}(f) d t \\
& \leq \frac{1}{2} \frac{\left(\frac{b}{a}\right)^{\alpha}-1}{\alpha} \bigvee_{b}^{b}(f)
\end{aligned}
$$

and

$$
\begin{aligned}
\left|\bar{\kappa}_{a+, b-}^{\alpha+\beta i} f-\frac{\left(\frac{b}{a}\right)^{\alpha+\beta i}-1}{(\alpha+\beta i)} \frac{f(b)+f(a)}{2}\right| & \leq \frac{1}{2} \int_{G(a, b)}^{b}\left(\frac{G(a, b)}{t}\right)^{\alpha} \frac{1}{t} \bigvee_{t}^{b}(f) d t+\frac{1}{2} \int_{a}^{G(a, b)}\left(\frac{t}{G(a, b)}\right)^{\alpha} \frac{1}{t} \bigvee_{a}^{t}(f) d t \\
& \leq \frac{1}{2} \frac{\left(\frac{b}{a}\right)^{\alpha}-1}{\alpha} \bigvee_{b}^{b}(f),
\end{aligned}
$$

where

$$
\bar{\kappa}_{a+, b-}^{\alpha+\beta i} f:=\frac{1}{2} \int_{G(a, b)}^{b}\left(\frac{t}{G(a, b)}\right)^{\alpha+\beta i} \frac{1}{t} f(t) d t+\frac{1}{2} \int_{a}^{G(a, b)}\left(\frac{G(a, b)}{t}\right)^{\alpha+\beta i} \frac{1}{t} f(t) d t .
$$

\section{References}

[1] A. Kilbas, H. M. Srivastava, J. J. Trujillo, Theory and Applications of Fractional Differential Equations, North-Holland Mathematics Studies, Elsevier Science B.V., Amsterdam, 2006.

[2] R. K. Raina, On generalized Wright's hypergeometric functions and fractional calculus operators, East Asian Math. J., 21 (2) (2005), 191-203.

[3] R. P. Agarwal, M.-J. Luo, R. K. Raina, On Ostrowski type inequalities, Fasc. Math., 56 (2016), 5-27.

[4] M. Kirane, B. T. Torebek, Hermite-Hadamard, Hermite-Hadamard-Fejer, Dragomir-Agarwal and Pachpatte type inequalities for convex functions via fractional integrals, Preprint arXiv:1701.00092. 
[5] S. S. Dragomir, Further Ostrowski and trapezoid type inequalities for the generalized Riemann-Liouville fractional integrals of functions with bounded variation, RGMIA Res. Rep. Coll., 20 (2017), Art. 84.

[6] A. Aglić Aljinović, Montgomery identity and Ostrowski type inequalities for Riemann-Liouville fractional integral, J. Math., 2014, Art. ID 503195, 6 pp.

[7] T. M. Apostol, Mathematical Analysis, Second Edition, Addison-Wesley Publishing Company, 1975.

[8] A. O. Akdemir, Inequalities of Ostrowski's type for $m$-and $(\alpha, m)$-logarithmically convex functions via Riemann-Liouville fractional integrals, J. Comput. Anal. Appl., 16(2) (2014), 375-383.

[9] G. A. Anastassiou, Fractional representation formulae under initial conditions and fractional Ostrowski type inequalities, Demonstr. Math., 48(3) (2015), 357-378.

[10] G. A. Anastassiou, The reduction method in fractional calculus and fractional Ostrowski type inequalities, Indian J. Math., 56(3) (2014), 333-357.

[11] H. Budak, M. Z. Sarikaya, E. Set, Generalized Ostrowski type inequalities for functions whose local fractional derivatives are generalized s-convex in the second sense, J. Appl. Math. Comput. Mech., 15(4) (2016), 11-21.

[12] P. Cerone, S. S. Dragomir, Midpoint-type rules from an inequalities point of view. Handbook of analytic-computational methods in applied mathematics, 135-200, Chapman \& Hall/CRC, Boca Raton, FL, 2000.

[13] S. S. Dragomir, The Ostrowski's integral inequality for Lipschitzian mappings and applications, Comput. Math. Appl., 38(11-12) (1999), 33-37.

[14] S. S. Dragomir, The Ostrowski integral inequality for mappings of bounded variation, Bull. Austral. Math. Soc., 60(3) (1999), 495-508.

[15] S. S. Dragomir, On the midpoint quadrature formula for mappings with bounded variation and applications, Kragujevac J. Math., 22 (2000), 13-19.

[16] S. S. Dragomir, On the Ostrowski's integral inequality for mappings with bounded variation and applications, Math. Ineq. Appl. 4 (2001), No. 1, 59-66. Preprint: RGMIA Res. Rep. Coll., 2 (1999), Art. 7.

[17] S. S. Dragomir, Refinements of the generalised trapezoid and Ostrowski inequalities for functions of bounded variation, Arch. Math., 91(5) (2008), 450-460.

[18] S. S. Dragomir, Refinements of the Ostrowski inequality in terms of the cumulative variation and applications, Analysis (Berlin) 34 (2014), No. 2, 223-240. Preprint: RGMIA Res. Rep. Coll., 16 (2013), Art. 29.

[19] S. S. Dragomir, Ostrowski type inequalities for Lebesgue integral: a survey of recent results, Australian J. Math. Anal. Appl., 14(1) (2017), 1-287.

[20] S. S. Dragomir, Ostrowski type inequalities for Riemann-Liouville fractional integrals of bounded variation, Hölder and Lipschitzian functions, Preprint RGMIA Res. Rep. Coll., 20 (2017), Art. 48.

[21] S. S. Dragomir, Ostrowski type inequalities for generalized Riemann-Liouville fractional integrals of functions with bounded variation, RGMIA Res. Rep. Coll., 20 (2017), Art. 58.

[22] A. Guezane-Lakoud, F. Aissaoui, New fractional inequalities of Ostrowski type, Transylv. J. Math. Mech., 5(2) (2013), 103-106

[23] A. Kashuri, R. Liko, Ostrowski type fractional integral inequalities for generalized ( $s, m, \varphi)$-preinvex functions, Aust. J. Math. Anal. Appl., 13(1) (2016), Art. 16, 11 pp.

[24] M. A. Noor, K. I. Noor, S. Iftikhar, Fractional Ostrowski inequalities for harmonic h-preinvex functions, Facta Univ. Ser. Math. Inform., 31(2) (2016), 417-445.

[25] M. Z. Sarikaya, H. Filiz, Note on the Ostrowski type inequalities for fractional integrals, Vietnam J. Math., 42(2) (2014), $187-190$.

[26] M. Z. Sarikaya, H. Budak, Generalized Ostrowski type inequalities for local fractional integrals, Proc. Amer. Math. Soc., 145(4) (2017), 1527-1538.

[27] E. Set, New inequalities of Ostrowski type for mappings whose derivatives are s-convex in the second sense via fractional integrals, Comput. Math. Appl., 63(7) (2012), 1147-1154.

[28] M. Tunç, On new inequalities for h-convex functions via Riemann-Liouville fractional integration, Filomat, 27(4) (2013), 559-565. 
[29] M. Tunç, Ostrowski type inequalities for $m$ - and $(\alpha, m)$-geometrically convex functions via Riemann-Louville fractional integrals, Afr. Mat., 27(5-6) (2016), 841-850.

[30] H. Yildirim, Z. Kirtay, Ostrowski inequality for generalized fractional integral and related inequalities, Malaya J. Mat., 2(3) (2014), 322-329.

[31] C. Yildiz, E, Özdemir, Z. S. Muhamet, New generalizations of Ostrowski-like type inequalities for fractional integrals, Kyungpook Math. J., 56(1) (2016), 161-172.

[32] H. Yue, Ostrowski inequality for fractional integrals and related fractional inequalities, Transylv. J. Math. Mech., 5(1) (2013), 85-89. 